\title{
Tonic Changes in EEG Power Spectra during Simulated Driving
}

\author{
Ruey-Song Huang, Tzyy-Ping Jung, and Scott Makeig \\ Swartz Center for Computational Neuroscience, Institute for Neural Computation, \\ University of California San Diego, La Jolla, CA, 92093-0961 USA \\ \{rshuang, jung, scott\}@sccn.ucsd.edu
}

\begin{abstract}
Electroencephalographic (EEG) correlates of driving performance were studied using an event-related lane-departure paradigm. High-density EEG data were analyzed using independent component analysis (ICA) and Fourier analysis. Across subjects and sessions, when reaction time to lanedeparture events increased several clusters of independent component activities in the occipital, posterior parietal, and middle temporal cortex showed tonic power increases in the delta, theta, and alpha bands. The strongest of these tonic power increases occurred in the alpha band in occipital and parietal regions. Other independent component clusters in the somatomotor and frontal regions showed less or no significant increase in all frequency bands as RT increased. This study demonstrates additional evidence of the close and specific links between cortical brain activities (via changes in EEG spectral power) and performance (reaction time) during sustained-attention tasks. These results may also provide insights into the development of human-computer interfaces for countermeasures for drowsy driving.
\end{abstract}

Keywords: EEG, ICA, driving, alertness, delta, theta, alpha, reaction time

\section{Introduction}

Drowsiness while driving is one of the major factors leading to crashes that result in severe injuries and fatalities [1-2]. Two of the basic rules of safe highway driving are to remain in the cruising lane and to keep an appropriate distance from other vehicles. Small changes in road curvature, uneven or slippery pavement, wind changes, or poor wheel alignment could make the vehicle drift out of the cruising lane. Lapses in attention and response to such lane drifts could result in collisions with other vehicles or run-off-road crashes. As drivers become fatigued and then drowsy, they exhibit slowed reaction time (RT) to traffic events, and increased deviation of vehicle lateral position (swerving) from lane center. Development of effective countermeasures to drowsy driving could prevent large numbers of serious accidents. Electroencephalography (EEG) is one of the most direct and effective physiological measures for assessing state of arousal. Several studies have demonstrated EEG correlates of fluctuations in performance during sustained attention tasks with characteristic time scales on the order of one second to several minutes [3-15]. These 
studies have suggested that low-frequency EEG power, particularly in the alpha (8-12 $\mathrm{Hz}$ ) and theta (4-7 Hz) bands, increase during periods of poor task performance (e.g., periods of high-error rate, lengthened RT, or failures to respond to driving challenges). In most studies, EEG power spectra were estimated from single-channel recordings at a few scalp sites, not allowing localization of the cortical sources of the observed EEG changes.

Our previous studies using independent component analysis (ICA) applied to high-density EEG data have demonstrated that an independent component (IC) with equivalent dipole sources located in the bilateral occipital cortex exhibits tonic changes in power spectral baseline highly correlated with performance fluctuation during sustained attention tasks, including simulated driving [12-15]. It is not known, however, whether the power spectra of other EEG processes are also strongly modulated by task performance. This study systematically explores tonic power spectral changes in the delta (1-3 Hz), theta (4-7 Hz), alpha (8-12 Hz), and beta (13$20 \mathrm{~Hz}$ ) bands in 13 independent component clusters of brain processes obtained across subjects and sessions during periods of increased reaction time to lanedeparture events during simulated driving.

\section{Materials and Methods}

\subsection{Experimental Paradigm and Participants}

A virtual-reality scene was created to simulate cruising in the fast lane of a straight highway at night. The driving simulator was implemented in $\mathrm{C} / \mathrm{C}++$ programming languages using the Open GL libraries on a desktop computer running the Linux operating system. During hour-long continuous driving sessions, computer-simulated lane-departure (deviation onset) events occurred every few seconds, during which the car drifted towards the curb or into the opposite lane with equal probability (Fig. 1). The vehicle did not 'crash' if the subject failed to respond but instead hit the virtual limit of the curb (after about $3 \mathrm{~s}$ of drift), and continued to move along the virtual curb until the subject resumed response by holding down an arrow key (response onset), and then releasing the key (response offset) when the car returned to the center of the cruising lane.

This paradigm was designed to assess subjects' responses to perturbing events embedded in continuous monotonous driving sessions, and to monitor continuous transitions from alertness to drowsiness [12-14]. Subjects' driving performance was measured by their reaction time (RT), defined as the duration between deviation onset and their response onset during each lane-departure event (trial). Slowed subject reaction times generally accompanied decreases in attention and alertness (Fig. 2).

Eleven right-handed healthy subjects with normal or corrected-to-normal vision participated in one or more hour-long sessions (20 sessions in all). All subjects gave informed consent before participating in an experimental protocol approved by the UCSD Human Research Protections Program. None of the subjects reported sleep deprivation the night before the experiment. Each subject had lunch about two hours 
before arriving at the lab around 2:00 PM; the driving experiment itself began near 3:00 PM after EEG cap and electrode set-up. During the experiment, the subject sat on a comfortable office chair with armrests $50 \mathrm{~cm}$ from a 19-inch monitor sitting in an EEG booth in which the background lighting was dim ( 2-3 lux).
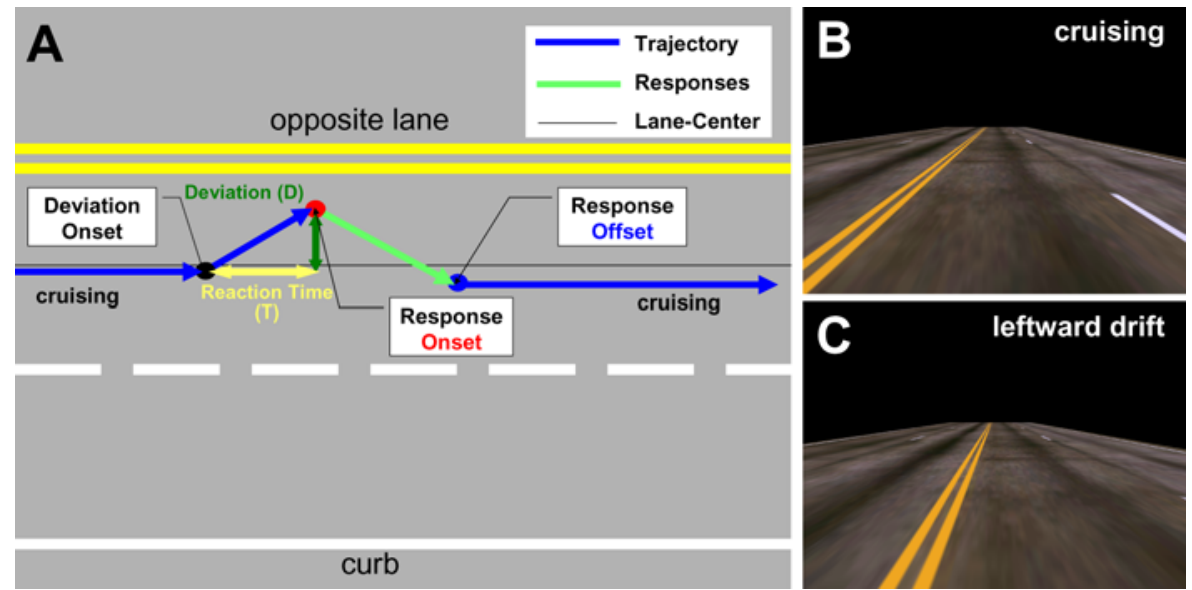

Fig. 1. Simulated driving experiment. A. Schematic diagram of the event-related lane departure paradigm (modified from a slide presented by R.-S.H. in [12]). B. A screen snapshot during cruising. C. A screen snapshot during a lane-departure event.

\subsection{Data Acquisition and Analysis}

256-channel EEG/EKG/EOG signals were recorded at $256 \mathrm{~Hz}$ using a BioSemi Active II acquisition system. Driving parameters (including lane positions, timing of event onsets and offsets) and subject behavioral responses were recorded at $256 \mathrm{~Hz}$ at the stimulus computer. A sequence of synchronized pulses was sent out from the PC parallel port to the BioSemi system for time stamping. The 3-D locations of all electrodes were digitized using a Polhemus system.

EEG data were digitally filtered using a linear FIR band pass filter $(1-45 \mathrm{~Hz})$ before further analysis. Continuous EEG time courses of all channels were segmented into 6-s epochs, from $1 \mathrm{~s}$ preceding to $5 \mathrm{~s}$ following deviation onsets. Subjects typically yawned or nodded a few times during hour-long sessions. These activities caused severe artifacts across all the channels in some epochs. Channels and epochs that contained severe artifacts, including extreme values of amplitudes, large linear trends, and abnormally distributed data (high kurtosis), were rejected semiautomatically before further analysis using functions of the open source EEGLAB toolbox [16] available at http://sccn.ucsd.edu/eeglab. Channels and epochs contaminated with other sources of artifacts (blinks, eye movements, cardiac activities, and persistent head-muscle noises) were not rejected, as these artifact sources could be separated from other EEG processes using ICA described below [1720]. 
The 6-s EEG epochs were concatenated into a two-dimensional matrix of size [channels, frames $\times$ epochs] after artifact rejection, and the matrix was reduced to 100 dimensions using Principle Component Analysis (PCA). Infomax ICA was applied to the dimension-reduced matrix, $x$, using the binica function with the 'extended' ICA option in EEGLAB. ICA finds an 'unmixing' matrix, $W$, which decomposes or linearly unmixes the matrix, $x$, into a sum of maximally temporally independent and spatially fixed components $u$, where $u=W x$. The rows of the output data matrix, $u$, are time courses of activations of the independent component (IC). The ICA unmixing matrix was trained separately for each session and subject. The initial learning rate was $10^{-4}$, and the training was stopped when the learning rate fell below $10^{-7}$.

To test cross-subject consistency of brain processes of interest, we grouped independent components obtained from multiple sessions and subjects semiautomatically into 13 IC clusters (Figs. 3 and 4) based on their scalp maps, dipole source locations, and mean power spectral baselines [15, 21-23]. The dipole sources locations were estimated according to the digitized 3-D electrode locations and ICA weight matrix for each session using the DIPFIT2 function in EEGLAB.

For each independent component, a logarithmic power spectral baseline was computed from a 1-s window before deviation onset in each 6-s epoch extracted from its activation time course using Fast Fourier Transform (FFT). For each component cluster, the logarithmic power spectral baselines of epochs from all subjects and sessions were grouped and sorted in ascending order by trial reaction time, resulting in a matrix of size [frequency bins $\times$ epochs]. The mean logarithmic power spectra of the first $10 \%$ of epochs below 3-s RT (periods of optimal performance) were subtracted from the matrix of RT-sorted power spectral baselines at each frequency bin. The normalized matrix was further subjected to moving average across RT-sorted epochs (trials) at each frequency bin using the same window size of the first $10 \%$ epochs below 3-s RT, with a step of 10 epochs. Mean tonic power changes in delta (1-3 Hz), theta (4-7 Hz), alpha (8-12 Hz), and beta (13-20 Hz) bands were obtained from the normalized and moving-averaged power spectral matrix (Figs. 3 and 4). A two-tailed t-test was used to assess if the mean power in each moving window was statistically different from that of the first $10 \%$ epochs below 3-s RT in each frequency band using a threshold of $\mathrm{p}<0.001$ corrected with a Bonferroni multiple comparison test.

\section{Results}

\subsection{Driving Performance as Measured by Reaction Time}

Across all sessions, subjects exhibited several fluctuations in their reaction times to lane-departure events. Some subjects became drowsy and hit the curb or drove into the opposite lane several times during hour-long sessions. Fig. 2A shows the fluctuation of subject reaction times in a representative session, where 666 lanedeparture events (trials) were recorded. Fig. 2B shows the same trials sorted by reaction time $(\mathrm{RT})$, which exhibit a 'bilinear' pattern (the majority of trial reaction 
times were short and increased exponentially in a small percentage of trials). The bilinear pattern was consistently observed across subjects and sessions, and on average $5 \%$ of trial reaction times were higher than $3 \mathrm{~s}$ (the approximate time lapsed before the vehicle hit the curb if the subject made no response).
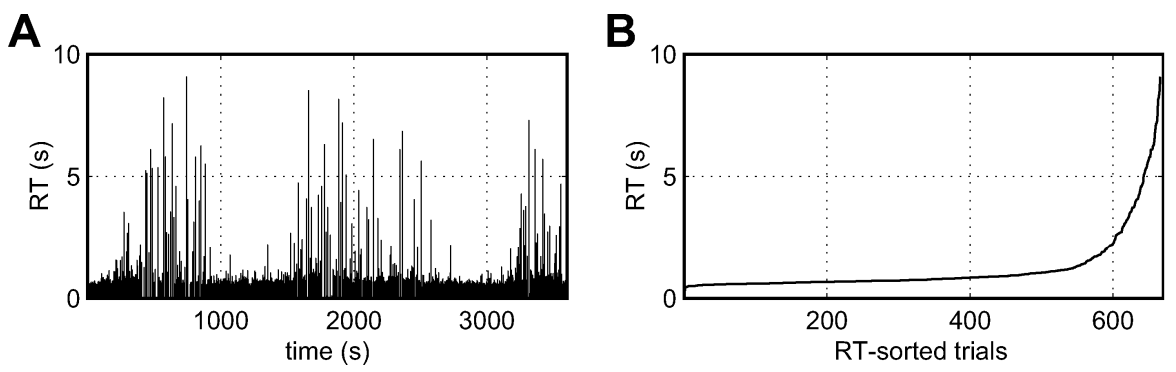

Fig. 2. Fluctuation of subject reaction times (RT) in a representative 1-hour session. A. Trial RTs in chronological order during the session. B. Trial RTs sorted in ascending order.

\subsection{Tonic Changes in Power Spectra in Relation to Reaction Time}

As reaction time increased, baseline power spectra of several independent component clusters in the occipital, parietal, and temporal regions showed significant increases relative to the mean power spectra of the first 10\% epochs (trials) below 3-s RT (Fig. $3)$. Other independent component clusters showed less significant or no tonic changes in their power spectra (Fig. 4). The observed tonic power changes in each frequency band are discussed below.

Delta Band Power (1-3 Hz). Mean baseline power in the delta band showed insignificant variations in short-RT $(<0.8 \mathrm{~s})$ epochs across all IC clusters. Mean delta band power started to increase as RT increased above $\sim 0.8 \mathrm{~s}$; these tonic changes were only significant in the bilateral occipital cluster (Fig. 2A) during protracted RTs $(>2 s)$.

Theta Band Power (4-7 Hz). Mean baseline power in the theta band showed similar changes as in the delta band power across all IC clusters. Mean theta band power remained unchanged or even decreased slightly as RT increased moderately $(<$ $0.8 \mathrm{~s}$ ), and started to increase at RTs above $\sim 0.8 \mathrm{~s}$. The tonic changes were only significant during protracted RTs (near or above $3 \mathrm{~s}$ ) in the bilateral occipital, medial posterior parietal, middle temporal, and somatomotor clusters (Figs. 3 and 4).

Alpha Band Power (8-12 Hz). Mean baseline power in the alpha band increased monotonically as RT increased in the bilateral occipital, medial posterior occipital, medial posterior parietal, and middle temporal clusters (Fig. 3). The tonic changes were significant at RTs above $\sim 0.8 \mathrm{~s}$ in the occipital and parietal clusters, and were stronger than the increases in the other frequency bands. In other IC clusters, as RT increased mean alpha band power showed both insignificant increases and decreases (Fig. 4).

Beta Band Power (13-20 Hz). As RT increased, mean baseline power in the beta band showed moderate increases in the bilateral occipital, medial posterior occipital, medial posterior parietal, and middle temporal clusters (Fig. 3). The tonic changes 
were significant at RTs above $\sim 0.8$-s in the bilateral occipital (Fig. 3A) and medial posterior parietal (Fig. 3D) clusters. As RT increased, mean beta band power showed insignificant changes in other IC clusters (Fig. 4).

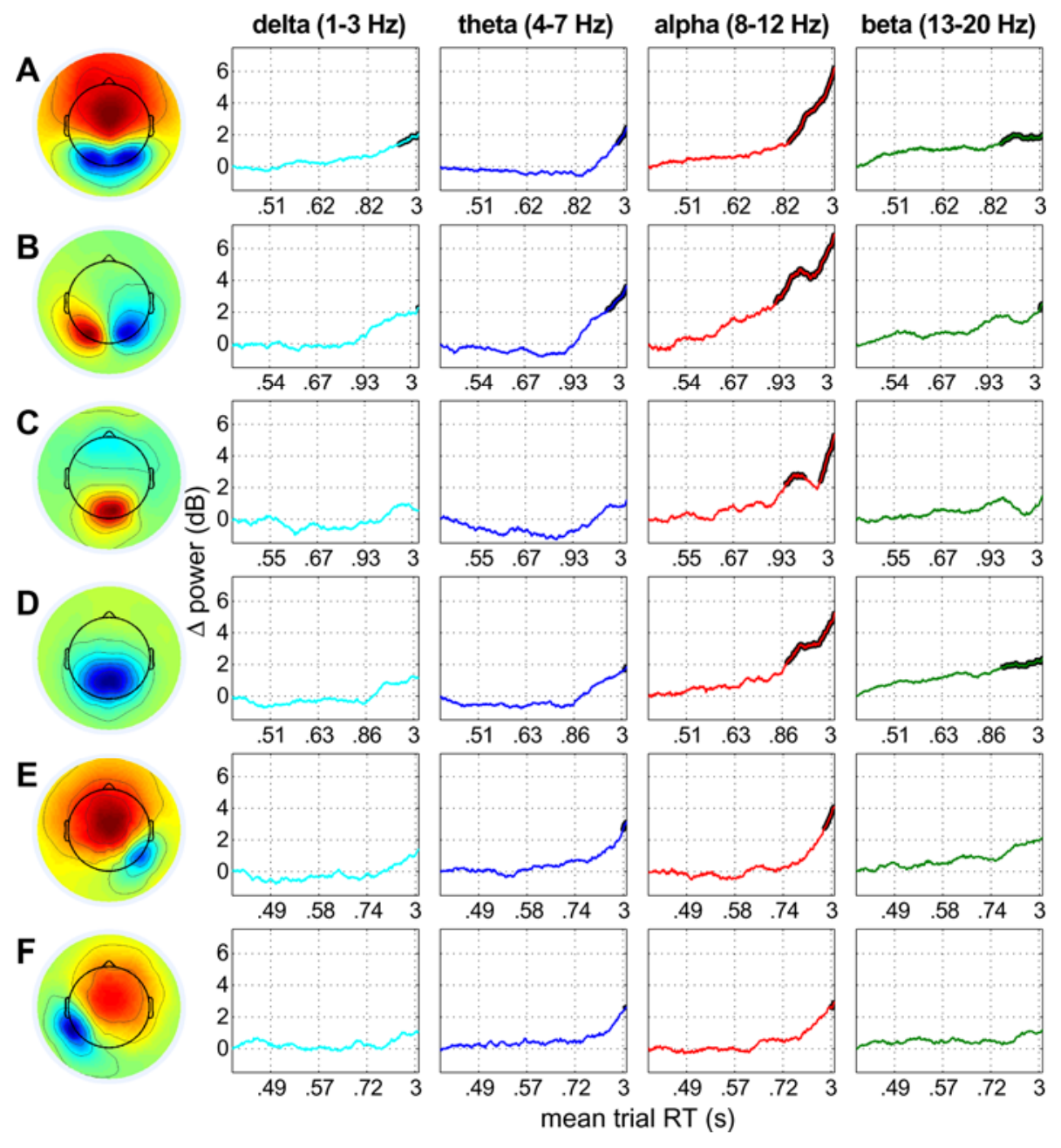

Fig. 3. Mean tonic power changes at four frequency bands for six independent component clusters. A. Bilaterally symmetric occipital cluster. B. Tangential occipital cluster. C. Medial posterior occipital cluster. D. Medial posterior parietal cluster. E. Right middle temporal cluster. F. Left middle temporal cluster. The scales of the vertical axes are the same in all subplots. The horizontal axis ticks include slow/drowsy (3-s) and 1st-3rd RT quartiles. Differences across clusters reflect differences in RT distribution in the 8-20 sessions of contributing components to each cluster. Color segments enclosed in thick black traces indicate significant $(\mathrm{p}<0.001$; corrected) tonic changes from the mean logarithmic power in the fastest $10 \%$ of epochs with RTs below 3-s. Note the significant changes in alpha band power in the occipital and parietal clusters. 


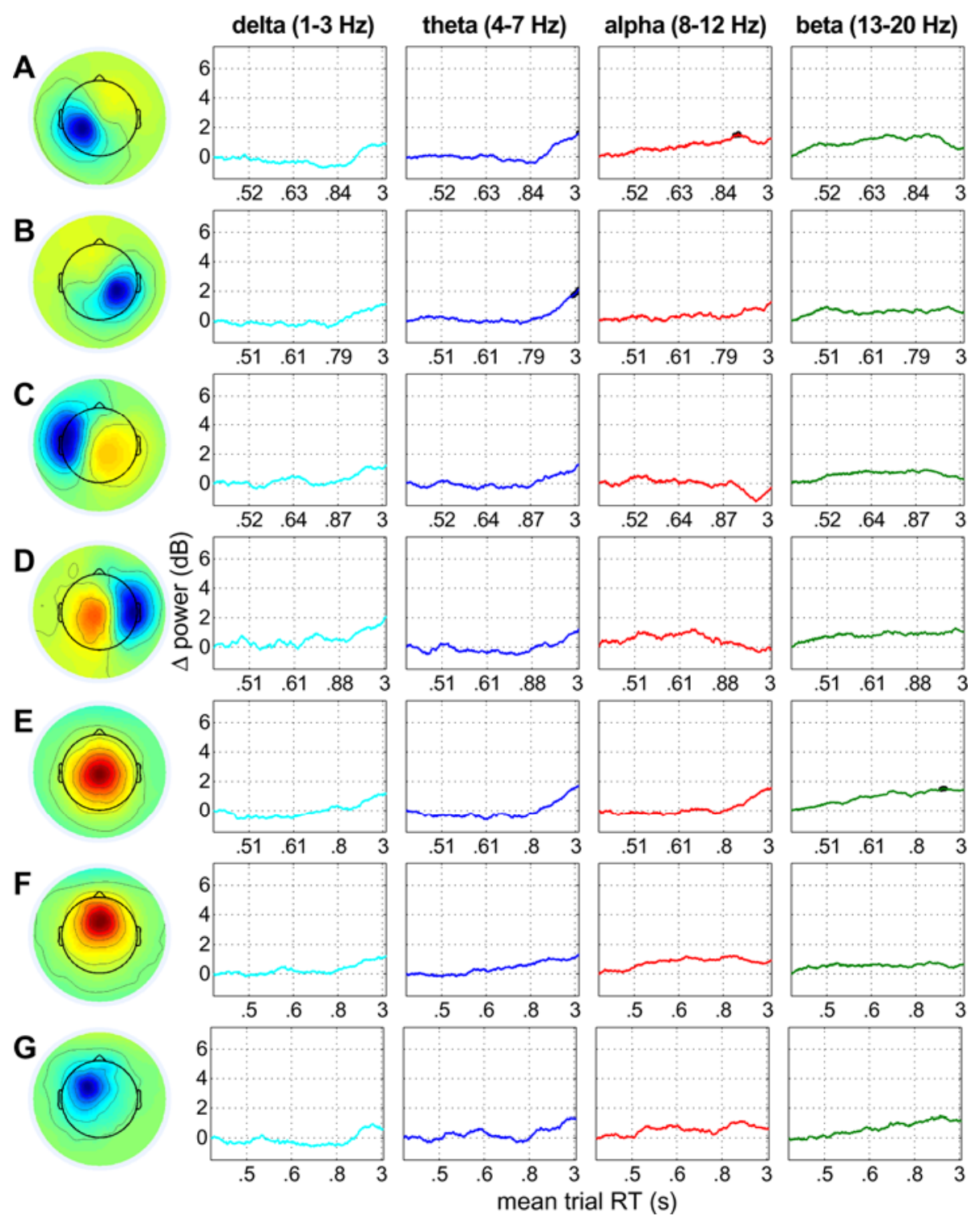

Fig. 4. Mean power in four frequency bands for seven independent component clusters showing no or weaker tonic power changes as RT increased. A. Left somatomotor cluster. B. Right somatomotor cluster. C. Left secondary somatosensory cluster. D. Right secondary somatosensory cluster. E. Central medial cluster. F. Frontal medial cluster. G. Left lateral frontal cluster. Other details as in Fig. 3. 


\section{Discussion}

In this study, we applied independent component analysis to dissociate multiple brain processes whose power spectra were modulated or unaffected by performance fluctuations during simulated driving. As subject reaction time to lane-departure events increased, several clusters of independent component activities in the occipital, posterior parietal, and middle temporal cortex showed tonic power increases in the delta, theta, and alpha bands across subjects and sessions. The power spectra of other independent component clusters in the somatomotor and frontal regions were less affected or were not affected by changes in reaction time. These results provide a more comprehensive insight into brain processes involved in sustained-attention tasks.

The event-related lane departure paradigm [12-14] used in this study may provide objective and quantitative measures of both instantaneous driving performance over shorter time spans (e.g., < $10 \mathrm{~s}$ ) and measures of average performance over longer periods (e.g., on the order of a minute). This paradigm has been replicated in other simulated driving experiments performed on a motion platform [24, 25]. Tonic power spectral changes in those experiments were similar to the results reported here; details will be reported elsewhere.

The strong tonic increases in alpha-band power in occipital and parietal regions likely index the gradual withdrawal of visuospatial attention as drowsiness increases [26]. These increases may also be used to predict reaction time in our task and very likely during simulated driving and real-life driving. Results of this study may also help guide development of EEG-based drowsiness detection and feedback systems, and may provide useful information for evaluating systems that directly detect and apply countermeasures to drowsy driving performance, such as lane departure warning systems (LDWS) and lane keeping assistance systems (LKAS) [27-30].

Acknowledgements. This research was supported by gifts from The Swartz Foundation (Old Field, NY). This work was also sponsored in part by the U.S. Defense Advanced Research Projects Agency (DARPA) under Grant NBCH1060010 and U.S. Army Research Laboratory under Grant W911NF-08-2-0003. The views and conclusions contained in this document are those of the authors and should not be interpreted as representing the official policies, either expressed or implied, of the DARPA, Army, or the U.S. Government. The U.S. Government is authorized to reproduce and distribute reprints for Government purposes notwithstanding any copyright notation heron. The authors would like to thank Julie Onton, Jennifer S. Kim, and Marisa Evans for help with experimental setup, and Arnaud Delorme for assistance with data analysis.

\section{References}

1. National survey of distracted and drowsy driving attitudes and behavior: 2002, volume 1 , DOT HS 809 566. http://www.nhtsa.dot.gov/people/injury/drowsy_driving1/surveydistractive03/index.htm 
2. Drowsy driving and automobile crashes, NCSDR/NHTSA Expert Panel on Driver Fatigue and Sleepiness. http://www.nhtsa.gov/people/injury/drowsy_driving1/Drowsy.html

3. Makeig, S., Inlow, M.: Lapses in alertness: coherence of fluctuations in performance and the EEG spectrum. Electroencephalogr. Clin. Neurophysiol. 86, 23-35 (1993)

4. Makeig, S., Jung, T.P.: Changes in alertness are a principal component of variance in the EEG spectrum. NeuroReport 7, 213-216 (1995)

5. Makeig, S., Jung, T.P.: Tonic, phasic and transient EEG correlates of auditory awareness in drowsiness. Cogn. Brain Res. 4, 15-25 (1996)

6. Jung, T.P., Makeig, S., Stensmo, M., Sejnowski, T.J.: Estimating alertness from the EEG power spectrum. IEEE Trans. Biomed. Eng. 44, 60-69 (1997)

7. Makeig, S., Jung, T.P., Sejnowski, T.J.: Awareness during drowsiness: dynamics and electrophysiological correlates. Canadian J. Exp. Psy. 54, 266-273 (2000)

8. Schier, M.A.: Changes in EEG alpha power during simulated driving: a demonstration. Int. J. Psychophysiol. 37, 155-162 (2000)

9. Huang, R.S., Tsai, L.L., Kuo, C.J.: Selection of valid and reliable EEG features for predicting auditory and visual alertness levels. Proc. Natl. Sci. Counc. Repub. China B Life Sci. 25, 17-25 (2001)

10. Lal, S.K., Craig, A.: Driver fatigue: electroencephalography and psychological assessment. Psychophysiol. 39, 313-321 (2002)

11. Lal, S.K., Craig, A.: Reproducibility of the spectral components of the electroencephalogram during driver fatigue. Int. J. Psychophysiol. 55, 137-143 (2005)

12. Huang, R.S., Jung, T.P., Duann, J.R., Makeig, S., Sereno, M.I.: Imaging brain dynamics during continuous driving using independent component analysis. In 35th Annual Meeting of the Society for Neuroscience, Washington D.C. (2005)

13. Huang, R.S., Jung, T.P., Makeig, S.: Multi-scale EEG brain dynamics during sustained attention tasks. Proc. IEEE ICASSP'07, vol. IV, 1173-1176 (2007)

14. Huang, R.S., Jung, T.P., Makeig, S.: Event-related brain dynamics in continuous sustained-attention tasks. In D.D. Schmorrow, L.M. Reeves (Eds.): Augmented Cognition, HCII 2007, LNAI 4565, 65-74 (2007)

15. Huang, R.S., Jung, T.P., Delorme, A., Makeig, S.: Tonic and phasic electroencephalographic dynamics during continuous compensatory tracking. NeuroImage 39, 1896-1909 (2008)

16. Delorme, A., Makeig, S.: EEGLAB: an open source toolbox for analysis of single-trial EEG dynamics including independent component analysis. J. Neurosci. Meth. 134, 9-21 (2004)

17. Bell, A.J., Sejnowski, T.J.: An information-maximization approach to blind separation and blind deconvolution. Neural Comput. 7, 1129-1159 (1995)

18. Lee, T.W., Girolami, M., Sejnowski, T.J.: Independent component analysis using an extended infomax algorithm for mixed subgaussian and supergaussian sources. Neural Comput. 11, 417-441 (1999)

19. Jung, T.P., Humphries, C., Lee, T.W., McKeown, M.J., Iragui, V., Makeig, S., Sejnowski, T.J.: Removing electroencephalographic artifacts by blind source separation. Psychophysiol. 37, 163-178 (2000)

20. Jung, T.P., Makeig, S., McKeown, M.J., Bell, A.J., Lee, T.W., Sejnowski, T.J.: Imaging brain dynamics using independent component analysis. Proc. IEEE 89, 1107-1122 (2001)

21. Makeig, S., Westerfield, M., Jung, T.P., Enghoff, S., Townsend, J., Courchesne, E., Sejnowski, T.J.: Dynamic brain sources of visual evoked responses. Science 295, 690-694 (2002)

22. Makeig, S., Delorme, A., Westerfield, M., Jung, T.P., Townsend, J., Courchesne, E., Sejnowski, T.J.: Electroencephalographic brain dynamics following manually responded visual targets. PLoS Biol. 2, 747-762 (2004) 
23. Onton, J., Westerfield, M., Townsend, J., Makeig, S.: Imaging human EEG dynamics using independent component analysis. Neurosci. Biobehav. Rev. 30, 808-822 (2006)

24. Lin, C.T., Wu, R.C., Jung, T.P., Liang, S.F., Huang, T.Y.: Estimating alertness level based on EEG spectrum analysis. EURASIP J. Appl. Signal Process. 19, 3165-3174 (2005)

25. Lin, C.T., Wu, R.C., Liang, S.F., Huang, T.Y., Chao, W.H., Chen, Y.J. Jung, T.P.: EEGbased drowsiness estimation for safety driving using independent component analysis. IEEE Trans. Circuit Sys. 52, 2726-2738 (2005)

26. Worden, M.S., Foxe, J.J., Wang, N., Simpson, G.V.: Anticipatory biasing of visuospatial attention indexed by retinotopically specific alpha-band electroencephalography increases over occipital cortex. J. Neurosci. 20, RC63 (2000)

27. Rimini-Doering, M., Altmueller, T., Ladstaetter, U., Rossmeier, M.: Effects of lane departure warning on drowsy drivers' performance and state in a simulator. In Proceedings of the Third International Driving Symposium on Human Factors in Driver Assessment, Training, and Vehicle Design, 88-95 (2005)

28. Kozak, K., Pohl, J., Birk, W., Greenberg, J., Artz, B., Bloomer, M., Cathey, L., Curry, R.: Evaluation of lane departure warnings for drowsy drivers. Proceedings of the Human Factors and Ergonomics Society 50th Annual Meeting, 2400-2404 (2006)

29. Navarro, J., Mars, F., Hoc, J.M.: Lateral control assistance for car drivers: a comparison of motor priming and warning systems. Hum. Factors 49, 950-960 (2007)

30. Kullack, A., Ehrenpfordt, I., Lemmer, K., Eggert, F., Reflekt, A.S.: lane departure prevention system based on behavioural control. IET Intell. Transp. Syst. 2, 285-293 (2008) 